\title{
Trastornos musculoesqueléticos y riesgos psicosociales de los técnicos de prevención de riesgos laborales
}

Study of the interrelationships between musculoskeletal disorders and psychosocial risk factors in occupational health and safety technicians

\section{Fermín Torrano}

${ }^{1}$ Universidad Internacional de La Rioja. Logroño, La Rioja, España

Fechas - Dates

Recibido: 2021.05 .24 Aceptado: 2021.05.24 Publicado: 2021.07.15
Correspondencia · Corresponding Author

Dr. Guillermo García González

Profesor Titular Derecho del Trabajo y SS Universidad Internacional de La Rioja (UNIR)

Email: guillermo.garcia@unir.net 


\section{Entrevista con el Dr. Fermín Torrano}

\section{1. ¿Cuál es la procedencia, especialización y áreas de trabajo de los autores del artículo? ¿Qué características le parecen más destacables de este grupo de trabajo?}

Los autores del trabajo pertenecen el grupo de investigación creado en el año 2017 en la Universidad Internacional de La Rioja denominado "Trabajo líquido y riesgos emergentes en la sociedad de la información- TRES-i:”. Este grupo reúne a un conjunto de profesionales de diferentes áreas y disciplinas académicas con el objetivo común de identificar y prevenir los riesgos emergentes en los nuevos modelos de organización empresarial basados en el uso de las tecnologías de la información y comunicación.

El interés del grupo está centrado en analizar, desde una perspectiva transversal y multidisciplinar, las nuevas formas de trabajo basadas en las TIC, haciendo especial énfasis en la repercusión que estas tienen sobre las notas básicas de la relación laboral y, singularmente, en la modulación del deber de protección empresarial. El análisis se centra en el trabajo líquido, tendencia que nace en la era de la información adaptándose a un mercado laboral cambiante. Junto a este propósito, el reto del grupo se dirige a identificar los riesgos emergentes en este nuevo contexto de relaciones laborales, elaborando propuestas prácticas que permitan su prevención, minimización y, en su caso, eliminación. En este sentido, se presentan como elementos fundamentales distintos riesgos laborales como la fatiga (visual, física y mental o psicológica), el estrés, el síndrome de estar quemado, las lesiones osteomusculares y la exposición laboral a nuevas sustancias químicas y agentes biológicos.

\section{2. ¿Cómo se ha financiado el estudio?}

Este estudio se ha financiado a través del Grupo de Investigación: "Trabajo líquido y riesgos emergentes en la sociedad de la información- TR3S-i, gracias al apoyo de la Universidad Internacional de la Rioja (UNIR), en el marco de su Programa de Proyectos Propios de Investigación UNIR 2018 [2018-2020].

\section{3. ¿Qué problema pretende abordar este estudio y dónde radica su interés o relevancia para la seguridad y salud en el trabajo?}

El objetivo principal de esta investigación es estudiar los principales factores de riesgo desencadenantes de los TME, aspecto que se ha convertido en las dos últimas décadas en uno de los principales focos de atención de la investigación en el ámbito del trabajo. A este respecto, se evidencia que existe una serie de aspectos psicosociales que predicen la aparición de molestias musculoesqueléticas, como el estrés, la excesiva carga y exigencia de trabajo, una baja satisfacción, el desequilibrio entre el esfuerzo realizado y las recompensas recibidas, la presión temporal o el escaso apoyo social por parte de jefes o compañeros, los cuales son elementos clave que influyen en el malestar y la frecuencia e intensidad de los síntomas físicos, como la fatiga y el dolor en espalda, manos y muñeca, aumentando la incidencia de los TME. 
Por otro lado, su interés radica en la población de referencia, técnicos de prevención, donde se observa la ausencia de estudios a nivel nacional e internacional que se hayan centrado en estudiar la existencia de posibles asociaciones entre TME y factores de riesgo psicosocial en estos trabajadores.

\section{4. ¿Qué aporta este estudio de novedoso o destacable en relación al resto de producción científica sobre el problema estudiado?}

Uno de los elementos más novedosos de la presente investigación ha sido la población de referencia, pues hasta ahora son muy pocos los estudios relacionados con los riesgos a los que se encuentran expuestos los trabajadores de este sector y, más específicamente, en relación a predecir las variables que mejor explican la aparición de molestias musculoesqueléticas.

En concreto, se detectó que una de las variables que mejor predicen la probabilidad de padecer molestias musculares es la formación en riesgos emergentes, además del género, la salud percibida y la exposición a riesgo psicosocial. La inclusión de esta variable supone otro aspecto también novedoso, pues la formación, a pesar de tener una relevancia muy significativa en el ámbito preventivo, es una variable que apenas se estudia en otras investigaciones.

\section{5. ¿Se han encontrado con alguna dificultad o contratiempo para el desarrollo del estudio?}

Una de las principales dificultades fue la ausencia de estudios en la población de los técnicos de prevención, lo cual impidió contrastar nuestros resultados con los obtenidos en la investigación previa. Por otro lado, otra de las limitaciones era conseguir una muestra suficientemente amplia para que las conclusiones tuvieran suficiente peso para ser consideradas relevantes y poder aumentar la sensibilización frente a los problemas que tienen estos profesionales. Afortunadamente, contamos con la colaboración de la Federación ASPA de Servicios de Prevención Ajenos, lo cual nos proporcionó una muestra lo suficientemente amplia para el estudio.

\section{6. ¿Se han obtenido los resultados esperados o se ha producido alguna sorpre- sa?}

Los resultados obtenidos están en consonancia con los obtenidos por los estudios previos realizados en el campo, aunque con otras muestras de trabajadores. En concreto, y en relación con las encuestas y estudios internacionales realizados por la Agencia Europea de Seguridad y Salud, una gran parte de estos trabajadores (técnicos de prevención) sufren habitualmente de dolencias musculares y exposición a riesgo psicosocial, aspectos muy relacionados con las condiciones en las que realizan su trabajo (uso de PVD, sobrecarga de trabajo, estrés, etc.).

En cuanto a la principal novedad que nos encontramos, queremos señalar el papel predictivo que juegan una serie de variables que apenas han recibido atención por parte de la investigación previa, como es la formación recibida en riesgos emergentes. Teníamos claro que la falta de formación era una variable que influía en 
gran medida, pero nos ha sorprendido la capacidad de amortiguación del riesgo que presenta.

\section{7. ¿Cómo ha sido el proceso de publicación? ¿Han sufrido alguna incidencia?}

El proceso de publicación ha sido el habitual, pues nuestro objetivo era publicar en una revista de cierto impacto para que el estudio tuviera la mayor difusión posible, así que apuntábamos a un JCR. Las normas, procesos de publicación y los tiempos han sido los habituales en este tipo revistas y no se ha producido ningún tipo de incidencia durante el mismo.

\section{8. ¿Qué implicaciones tiene este estudio para la prevención de riesgos labora- les? ¿Cuál sería la recomendación para mejorar la práctica profesional en rela- ción al problema estudiado?}

A nuestro juicio, el desarrollo de estrategias eficaces para prevenir y gestionar de manera efectiva la posible aparición de TME relacionados con el trabajo parte de la necesidad de comprender la naturaleza multifactorial de su desarrollo etiológico. Además, hay evidencias que sugieren que las intervenciones con un enfoque concentrado en los problemas de organización del trabajo tienen el potencial de reducir el estrés laboral y, a su vez, los síntomas del cuello y las extremidades superiores.

En definitiva, los hallazgos del presente estudio han demostrado la relación existente entre la exposición a riesgos físicos y psicosociales y la presencia de diferentes TME, lo que aumenta la necesidad de evaluar de forma conjunta ambos aspectos. En concreto, el género, la falta de formación y de pausas, una autopercepción negativa en relación con la salud y la valoración profesional en el trabajo y un elevado riesgo psicosocial, resultaron aspectos clave a la hora de explicar la probabilidad de sufrir TME.

\section{Resumen $^{(1)}$}

El propósito de esta investigación es examinar la presencia de trastornos musculoesqueléticos (TME) y exposición a riesgo psicosocial en una muestra de 399 Técnicos de prevención, profundizando en las asociaciones entre ambos aspectos. Para ello, se hizo uso del Cuestionario Nórdico Estandarizado y el Cuestionario Decore. Con los datos obtenidos, se llevaron a cabo distintos análisis descriptivos, correlacionales y un modelo de análisis multivariante. El 77,17\% de los participantes afirmó haber padecido alguna dolencia muscular, lo que se relaciona con el género, las horas semanales de trabajo de oficina y la formación en riesgos emergentes. Los factores de riesgo psicosocial más críticos son los relacionados con las recompensas que obtiene el trabajador por su trabajo, con el 54,7\% de los técnicos en situación de alerta o emergencia, y con las exigencias y requerimientos cognitivos de las tareas, con el 38,8\% en un estado de alerta. Se destaca que el hecho de no sentirse valorado profesionalmente, trabajar fuera del horario laboral, tener molestias musculoesqueléticas y percibir un estado de salud mala o regular, aumentan la puntuación del IGR. El estudio resalta la alta exposición de 
los técnicos de prevención a factores de riesgo tanto físicos como psicosociales y la relación significativa entre la prevalencia de dolencias musculares y los factores de riesgo psicosocial en estos trabajadores. Además, se ha establecido un modelo predictivo del sufrimiento de malestar muscular concretando las variables que mejor predicen la probabilidad de padecerlo: género, formación en riesgos emergentes, salud percibida y exposición al riesgo psicosocial.

\section{Referencias}

1. López-González MJ, González-Menéndez E, González S, Torrano F. Study of the interrelationships between musculoskeletal disorders and psychosocial risk factors in occupational health and safety technicians. International Journal of Occupational Safety and Ergonomics. 2021. doi:10.1080/10803548.2021.1902137 